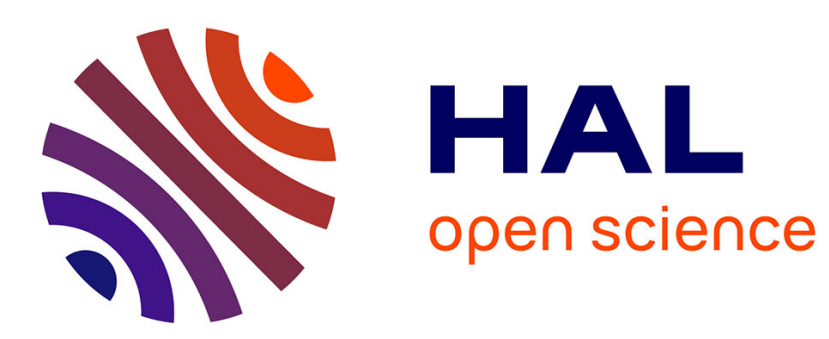

\title{
Robust estimation of the cerebral blood flow in arterial spin labelling
}

Camille Maumet, Pierre Maurel, Jean-Christophe Ferré, Christian Barillot

\section{To cite this version:}

Camille Maumet, Pierre Maurel, Jean-Christophe Ferré, Christian Barillot. Robust estimation of the cerebral blood flow in arterial spin labelling. Magnetic Resonance Imaging, 2014, 32 (5), pp.497 - 504. 10.1016/j.mri.2014.01.016 . inserm-00942814

\section{HAL Id: inserm-00942814 https://www.hal.inserm.fr/inserm-00942814}

Submitted on 19 Mar 2014

HAL is a multi-disciplinary open access archive for the deposit and dissemination of scientific research documents, whether they are published or not. The documents may come from teaching and research institutions in France or abroad, or from public or private research centers.
L'archive ouverte pluridisciplinaire HAL, est destinée au dépôt et à la diffusion de documents scientifiques de niveau recherche, publiés ou non, émanant des établissements d'enseignement et de recherche français ou étrangers, des laboratoires publics ou privés. 


\title{
Robust Estimation of the Cerebral Blood Flow in Arterial Spin Labelling
}

\author{
Camille Maumet ${ }^{\mathrm{a}, \mathrm{b}, \mathrm{c}, \mathrm{d}, *}$, Pierre Maurel $\mathrm{l}^{\mathrm{a}, \mathrm{b}, \mathrm{c}, \mathrm{d}}$, Jean-Christophe Ferré Me,b,c,d,e, $^{\text {, }}$ \\ Christian Barillot ${ }^{\mathrm{a}, \mathrm{b}, \mathrm{c}, \mathrm{d}}$, \\ ${ }^{a}$ University of Rennes 1, Faculty of medecine, F-35043 Rennes, France \\ ${ }^{b}$ INSERM, U746, F-35042 Rennes, France \\ ${ }^{c}$ CNRS, IRISA, UMR 6074, F-35042 Rennes, France \\ ${ }^{d}$ Inria, VISAGES project-team, F-35042 Rennes, France \\ ${ }^{e}$ CHU Rennes, Department of Neuroradiology, F-35033 Rennes, France
}

\begin{abstract}
The introduction of Arterial Spin Labelling (ASL) techniques in Magnetic Resonance Imaging (MRI) has made feasible a non-invasive measurement of the Cerebral Blood Flow (CBF). However, to date, the low signal-to-noise ratio of ASL gives us no option but to repeat the acquisition to accumulate enough data in order to get a reliable signal. The perfusion signal is then usually extracted by averaging across the repetitions. But the sample mean is very sensitive to outliers. A single incorrect observation can therefore be the source of strong detrimental effects on the perfusion-weighted image estimated with the sample mean.

We propose to estimate robust ASL CBF maps with M-estimators to overcome the deleterious effects of outliers. The behaviour of this method is compared to z-score thresholding as recommended in [1]. Validation on simulated and real data is provided. Quantitative validation is undertaken by measuring the correlation with the most widespread technique to measure perfusion with MRI: Dynamic Susceptibility weighted Contrast imaging.
\end{abstract}

Keywords: Arterial Spin Labelling, M-estimators, Robust statistics

\footnotetext{
${ }^{*}$ Corresponding author

Email address: contact@camillemaumet.com (Camille Maumet)
} 


\section{Introduction}

Arterial Spin Labelling (ASL) allows a non-invasive quantification of the Cerebral Blood Flow (CBF) [2]. Due to the low Signal-to-Noise Ratio (SNR) of the ASL sequence, a single pair of control and label image is not sufficient to measure perfusion. The acquisition is therefore repeated several times, leading to $r$ pairs of images (usually $r \geq 30$ ). Perfusion information is then usually extracted by pair-wise subtracting the control and label images (or using surround subtraction in functional ASL) [3] and averaging across the repetitions $[4,5]$.

Though sample mean, as an unbiased estimate of mean, ensures convergence as $r$ grows, it has a breakdown point of $0 \%$ (i.e. a single arbitrary large value can induce an arbitrary large estimate) and is thus very sensitive to outliers as illustrated in fig. 1. And yet it is well-known that instabilities

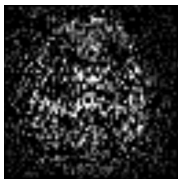

Repeat 1

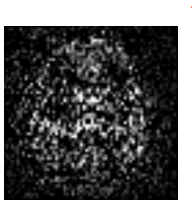

Repeat 1
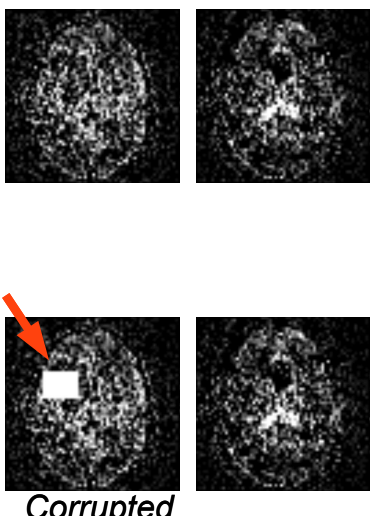

repeat

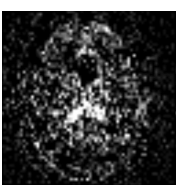

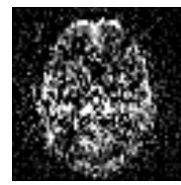

Repeat $\mathrm{n}$

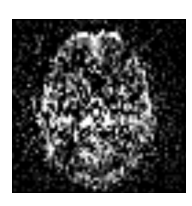

Repeat $\mathrm{n}$

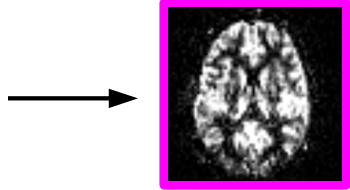

ASL CBF (average)

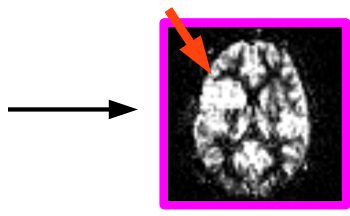

ASL CBF

(average)

Figure 1: Sensitivity of the sample mean to outliers. A single corrupted volume can induce strong artefact in the final CBF maps computed with the sample mean (red arrow).

during the acquisition and improperly corrected patient motion can cause artefactual values [6]. In particular, sudden subject motion often induces strong corolla-shaped artefacts [7].

To avoid the detrimental effects that a few abnormal repetitions could have in the final perfusion map, it is often suggested to ignore the volumes corresponding to the motion peaks using an appropriate threshold [7]. Volumes with (estimated) motion parameters greater than $[1-3]^{\circ}$ or $[1-3] \mathrm{mm}$ are thus discarded before averaging. However the choice of these thresh- 


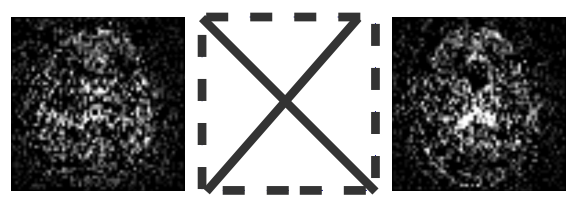

Repeat 1

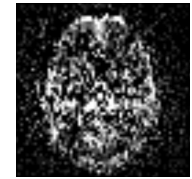

Repeat $n$

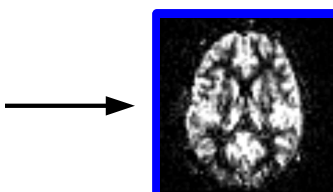

ASL CBF

(Z-score thresh.)

Figure 2: Volume exclusion via z-score thresholding as proposed in [1].

olds is empirical and there is no common rule across studies or automatic methods to tune these ad-hoc parameters. In [1], the authors proposed an automatic algorithm for outlier rejection in ASL perfusion series based on zscore thresholding at the volume (or slice) level as illustrated in fig. 2. Their method produced satisfactory results on a qualitative validation based on ratings made by medical experts. However, their approach is based on zscores, while more robust statistical measures are known to be better suited to deal with outliers. Also, they rely on empirically tuned parameters that might limit the generalisation of their procedure to new datasets.

How to appropriately deal with outliers has been widely studied in the statistical literature and a large range of methods has emerged. Z-score is known to be sensitive to sample size and is suffering from masking effects when more than one outliers is present in the series [8]. Indeed, in a dataset containing more than one outlier, the standard deviation estimate will be artificially inflated which may prevent z-score based outlier detection. On the other hand, M-estimators are robust techniques to estimate location and scale in the presence of outliers [9]. We focus on Huber's M-estimator [10], as it is the most widely used. An example of estimate computed with this method is provided in fig. 3 .

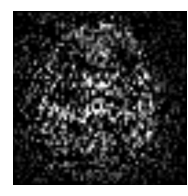

Repeat 1

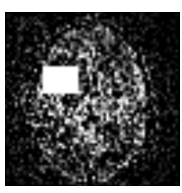

Corrupted repeat

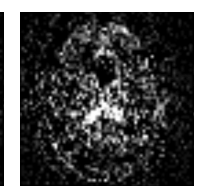

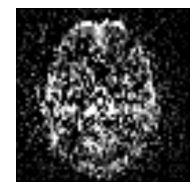

Repeat $n$

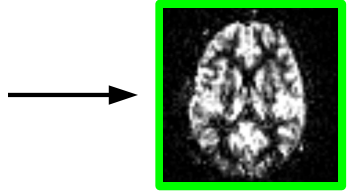

ASL CBF (Huber M-est.)

Figure 3: Robust ASL CBF map via Huber's M-estimator.

In this paper, we propose to estimate robust ASL CBF maps with $\mathrm{Hu}$ - 
ber's M-estimator. This method is compared to z-thresholding as proposed in [1]. Validation is undertaken by measuring the voxel-to-voxel correlation between ASL CBF maps and CBF maps computed from Dynamic Susceptibility weighted Contrast (DSC) imaging as an affine relationship is expected between these estimates of CBF [11].

Part of this work was published in the Multimodal Brain Image Analysis workshop in 2012 [12]. In this paper, the methods is presented in greater details and a new validation on real clinical datasets is provided.

Section 2 presents the theory underlying the statistical methods. Section 3 describes the validation procedure and the datasets under study. Section 4 presents the results on simulated data and on real datasets from patients diagnosed with brain tumours.

\section{Theory}

Starting from a perfusion-weighted series, namely a 4D volume made of the $r$ repetitions obtained after pair-wise subtracting the control and label scans, the objective is to compute a single perfusion-weighted volume. This section presents z-score thresholding in 2.1 and M-estimators in 2.2 as statistical methods to compute robust CBF maps.

\subsection{Z-score thresholding}

In [1], an outlier rejection algorithm based on z-scores was proposed in order to remove outliers from the perfusion-weighted series. The outlier rejection is performed both on a volume-by-volume and a slice-by-slice basis. For each volume (respectively slices) $v$, the mean $m_{v}$ and standard deviation $s_{v}$ of in-brain voxel intensities is computed. Assuming a Gaussian distribution of $m_{v}$ and $s_{v}$, a volume is then rejected if:

$$
\begin{array}{r}
\left|m_{v}\right|>\hat{\mu}_{m}+2.5 \hat{\sigma}_{m} \text {, or } s_{v}>\hat{\mu}_{s}+1.5 \hat{\sigma}_{s} \\
\text { where } \hat{\mu}_{m}=\frac{1}{r} \sum_{i=1}^{r} m_{i} \hat{\sigma}_{m}^{2}=\frac{1}{r-1} \sum_{i=1}^{r}\left(m_{i}-\hat{\mu}_{m}\right)^{2} \\
\text { and } \hat{\mu}_{s}=\frac{1}{r} \sum_{i=1}^{r} s_{i} \hat{\sigma}_{s}^{2}=\frac{1}{r-1} \sum_{i=1}^{r}\left(s_{i}-\hat{\mu}_{s}\right)^{2} .
\end{array}
$$

The parameters 1.5 and 2.5 were determined empirically. To avoid overfiltering, a heuristic is added saying that series verifying:

$$
\ln \left(\max _{i}\left(s_{i}\right)-\min _{i}\left(s_{i}\right)\right)<1
$$


are not searched for outliers. Once the outliers are identified, the perfusion map is then computed by averaging the remaining repetitions voxel-by-voxel.

\subsection{M-estimators}

A theoretically more efficient approach to deal with outliers is to employ robust statistics, such as M-estimators. In [10], M-estimators are defined, given a function $\rho$, as solutions $\hat{\theta}$ of:

$$
\hat{\theta}=\underset{\theta}{\operatorname{argmin}}\left(\sum_{i=1}^{r} \rho\left(x_{i}-\theta\right)\right) .
$$

If $\rho$ is differentiable, and $\psi$ is its derivative then eq. (3) can be solved by finding the root of:

$$
\sum_{i=1}^{r} \psi\left(x_{i}-\theta\right)=0
$$

The sample mean can be seen as an M-estimator with $\rho\left(x_{i}-\theta\right)=\left(x_{i}-\theta\right)^{2}$ and $\psi\left(x_{i}-\theta\right)=2\left(x_{i}-\theta\right)$ leading to $\hat{\theta}=\frac{1}{r} \sum_{i=1}^{r} x_{i}$.

The M-estimator of location proposed by Huber in [10] is defined by:

$$
\psi\left(x_{i}-\theta\right)=\gamma\left(\frac{x_{i}-\theta}{\sigma}\right) \quad \text { where } \gamma(x)=\left\{\begin{array}{rr}
-k, & x<-k, \\
x, & -k<x<k, \\
k, & x>k .
\end{array}\right.
$$

$k$ will be set to 1.345 throughout this paper corresponding to $95 \%$ efficiency in Gaussian data [13]. Likewise, $\sigma$ is estimated by a robust estimator: the median absolute deviation divided by 0.6745 [9]. Huber's M-estimator is applied voxel by voxel on the perfusion-weighted series to obtain the robust perfusion-weighted map. Fig. 4 illustrates the $\psi(x)$ functions for the three estimators of interest. The $\psi$ function for z-score thresholding is equivalent to the $\psi$ function of the sample mean applied to the data after outlier removal.

\section{Material and Methods}

\subsection{Data}

\subsubsection{Acquisition protocol}

26 patients diagnosed with brain tumours were involved in this study. Two patients were excluded because of strong borderzone signs [14]. The 


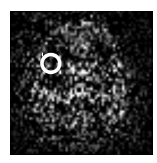

Repeat 1

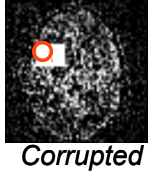

repeat

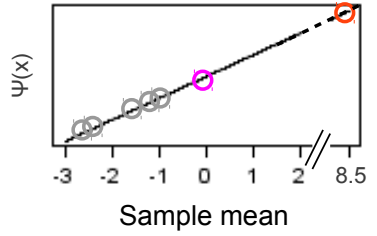

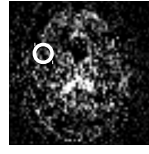
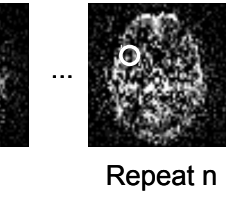

Repeat $n$

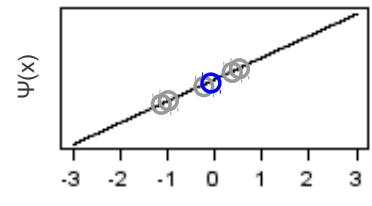

Z-score thresholding (sample mean after outlier rejection)

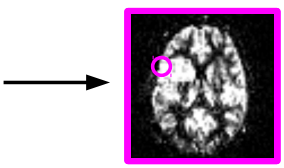

Sample mean Z-score thresh. Huber M-est.

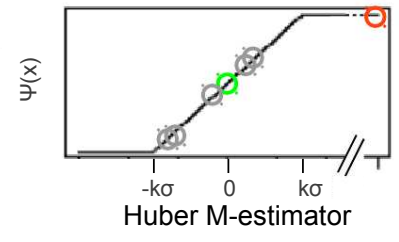

Figure 4: $\psi$ functions for the three estimators: sample mean, z-score thresholding (equivalent to a sample mean applied after outlier rejection) and Huber's M estimator.

final dataset therefore included 24 patients (15 males, 9 females, age: 55.3 \pm 16.2 years). Data acquisition was performed on a $3 \mathrm{~T}$ Siemens Verio Magnetic Resonance (MR) scanner with a 32-channel head-coil in the context of clinical practice. The imaging protocol included a 3D T1-weighted anatomical sequence (TR: $1900 \mathrm{~ms}$, TE: $2.27 \mathrm{~ms}$, FOV: $256 \mathrm{~mm}$ x $256 \mathrm{~mm}$ x $176 \mathrm{~mm}$, flip angle: $9^{\circ}$, resolution: $1 \mathrm{~mm} \times 1 \mathrm{~mm} \times 1 \mathrm{~mm}$ ), a PICORE Q2TIPS sequence with crusher gradients (TR: $3000 \mathrm{~ms}$, TE: $18 \mathrm{~ms}$, FOV: $192 \mathrm{~mm}$ x $192 \mathrm{~mm}$, flip angle: 90 , in plane resolution: $3 \mathrm{~mm}$ x $3 \mathrm{~mm}$, slice thickness: $7 \mathrm{~mm}$, inter-slice gap: $0.7 \mathrm{~mm}$, TI: $1700 \mathrm{~ms}$, bolus width: $700 \mathrm{~ms}$, $r=60$ ), a DSC sequence (GRE EPI, TR: $1500 \mathrm{~ms}$, TE: $30 \mathrm{~ms}$, FOV: $230 \mathrm{~mm}$ x $230 \mathrm{~mm}$, flip angle: $90^{\circ}$, in plane resolution: $1.8 \mathrm{~mm}$ x $1.8 \mathrm{~mm}$, slice thickness: $4 \mathrm{~mm}$, inter-slice gap: $1.2 \mathrm{~mm}$ ) and 3D T1-weighted post gadolinium sequence (TR: $1900 \mathrm{~ms}$, TE: $2.27 \mathrm{~ms}$, flip angle: 9, FOV: $250 \mathrm{~mm}$ x $250 \mathrm{~mm}$ x $176 \mathrm{~mm}$, resolution: $1 \mathrm{~mm} \times 1 \mathrm{~mm} \times 1 \mathrm{~mm}$ ).

1 healthy subject was involved in this study. The imaging protocol included a 3D T1-weighted anatomical sequence (same parameters as above) and a PICORE Q2TIPS sequence with crusher gradients (TR: $2500 \mathrm{~ms}$, TE: $19 \mathrm{~ms}$, flip angle: $90^{\circ}$, in plane resolution: $3 \mathrm{~mm} \mathrm{x} 3 \mathrm{~mm}$, slice thickness: $7 \mathrm{~mm}$, inter-slice gap: $0.7 \mathrm{~mm}$, TI: $1800 \mathrm{~ms}$, bolus width: $700 \mathrm{~ms}$, $r=250)$. 


\subsubsection{Pre-processing}

Image pre-processing was performed using SPM8 ${ }^{1}$ (Statistical Parametric Mapping 8, Wellcome Department of Imaging Neuroscience, University College, London) Matlab toolbox. A six-parameter rigid-body registration of the ASL volumes was carried out in order to reduce undesired effects due to subject motion. Coregistration on grey matter map was then performed based on normalised mutual information.

The unlabelled and labelled ASL volumes were pair-wise subtracted in order to obtain a perfusion-weighted series per subject. Estimation of robust ASL perfusion-weighted maps was then carried out as described in section 3. A standard kinetic model [15] was then applied in order to obtain quantitative ASL CBF maps.

The DSC images were processed using MR manufacturer software by manually choosing an arterial input function to calculate $\mathrm{CBF}$ and mean transit time maps based on a deconvolution algorithm [16]. Similarly to ASL, DSC CBF maps were coregistered on grey matter maps.

\subsection{Validation}

In order to compare the three approaches, the sample mean, z-score thresholding and Huber's M-estimator, we performed a validation on data with simulated corruption, as described in 3.2.1 and on real clinical datasets as presented in 3.2.2.

\subsubsection{Simulated corruption}

In order to assess the efficiency of each technique, we generated simulated data with a known quantity of outliers based on two real datasets. Outliers were drawn from a uniform distribution with extrema $(-100 ; 100)$. These values were determined empirically. Indeed, in an uncorrupted perfusionweighted map, we observed values ranging between -10 and +10 with standard deviations up to 50. Also, by looking at the values of identified outliers in a real dataset, we found values as big as 300 in absolute value.

As data corruption usually affects multiple voxels per volume [7, 1], outlier simulation was undertaken by corrupting from $0 \%$ (not corrupted) to $50 \%$ of the volumes. We will lately refer to these corrupted volumes as outlier volumes. Then, $2 \%, 20 \%$ or $50 \%$ of the voxels in each outlier volume were

\footnotetext{
${ }^{1}$ http://www.fil.ion.ucl.ac.uk/spm/
} 
replaced by random outliers leading to low, medium and high level of volume corruption respectively. A brief overview of the simulated data corruption process is provided in fig. 5. Each simulation was repeated 30 times in order to get estimates of the standard error. Simulated data were based on two real datasets as described hereafter.

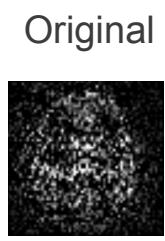

Repeat 1

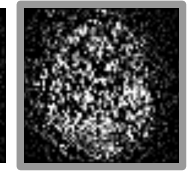

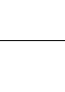

\section{For each corrupted volume}

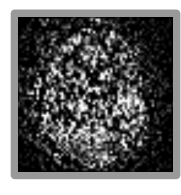

Repeat i

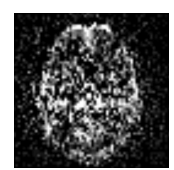

Repeat $r$
Series with simulated corruption

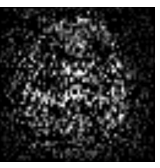

Repeat 1

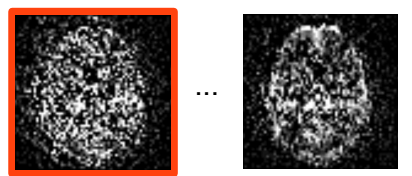

Repeat $r$

Figure 5: Simulation: corruption of a given percentage of ASL volumes (from 0\% (not corrupted) to $50 \%$ ) by adding samples drawn from a uniform distribution to a given percentage of the voxels $(2 \%, 20 \%$ or $50 \%)$ per volume.

The first dataset was a perfusion-weighted series with a large number of repetitions, $r=250$, from a healthy subject. The 60 first volumes of the series (obtained after pair-wise subtraction) were extracted, corrupted by simulation and then used as dataset for robust CBF map estimation. The perfusion-weighted map obtained by averaging the 250 repetitions was used as the ground truth. The quality of the maps produced by each method was measured in term of sum of squared differences with the ground truth.

The second dataset was a perfusion-weighted series of one of the patients diagnosed with a brain tumour. The original ASL CBF map of this subject presented few artefacts identified by visual inspection and a very low level of motion ( $<0.5 \mathrm{~mm}$ and $<0.2^{\circ}$ in all directions). The 60 volumes of the series were corrupted by simulation and then used as dataset for robust CBF map estimation. As DSC is currently the reference method to estimate perfusion with Magnetic Resonance Imaging (MRI), the quality of the maps 
produced by each method was measured by computing the Pearson linear correlation coefficient with the DSC CBF map. The choice of a correlation metric is supported by the fact that an affine relationship can usually be assumed between CBF maps produced by ASL and DSC [11].

\subsubsection{Experiments on real clinical data sets}

The performances of the sample mean, z-score thresholding and Huber's M-estimator were also compared on real clinical datasets.

Full dataset comparison. For each of the 26 patients involved in this study, an ASL CBF map was estimated based on the full perfusion-weighted series. This is the procedure that would be applied as a pre-processing step in a standard ASL study. For validation purposes, DSC was used to estimate a ground truth CBF map. The quality of the maps produced by each method based on ASL data, was then measured by computing the Pearson linear correlation coefficient with the DSC CBF map.

Comparison based on subsets of the available data. To investigate the behaviour of the different $\mathrm{CBF}$ estimators on datasets of various sizes, we further decimated the data (to get from 5 to 50 volumes) by randomly selecting a subset of the volumes in the perfusion-weighted series of each subject. The experiment was repeated 20 times. Again, the performances were assessed by computing the Pearson linear correlation coefficient with the DSC CBF maps.

\section{Results}

In this section, we present the results of the validation on data corrupted by simulation in 4.1 and on real clinical datasets in 4.2

\subsection{Validation on simulated data}

\subsubsection{Dataset with 250 repetitions:}

Fig. 6 presents the simulation study based on the first dataset, obtained from a healthy subject. The performances of sample mean, z-score thresholding [1], and Huber's M-estimator are assessed by measuring the sum of squared differences of the ASL CBF map with the ground truth estimated by averaging a large number of repetitions.

As described in fig. 6, with a medium or a high level of corruption, zscore thresholding and Huber's M-estimator perform equally and better than 

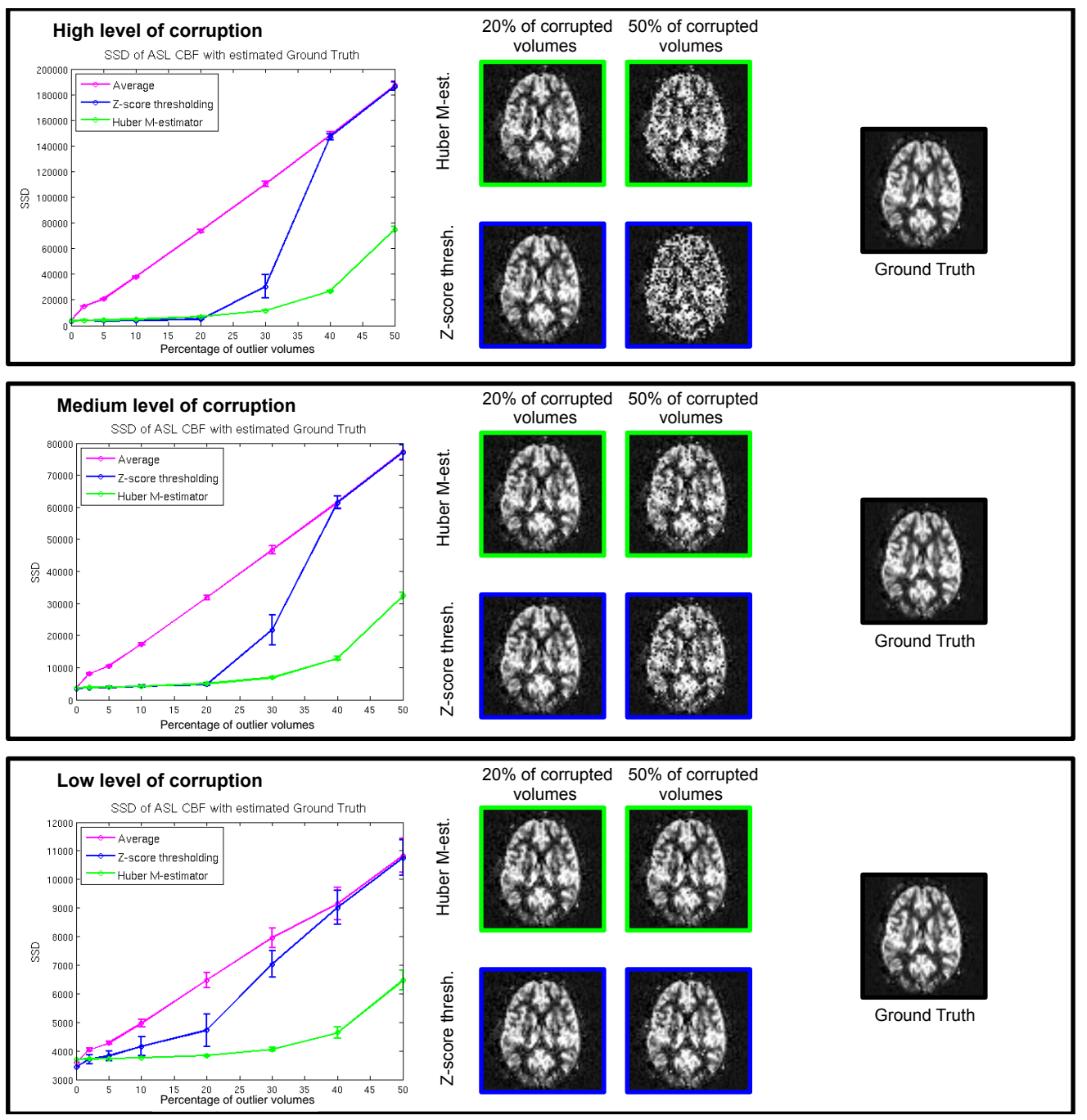

Figure 6: Healthy subject dataset with simulated outliers: sum of squared differences (SSD) of ASL CBF map, computed by M-estimator, z-score thresholding [1] and sample mean, with the estimated ground truth. High, medium and low level of volume corruption, from $0 \%$ to $50 \%$ of corrupted volumes. In all configuration Huber's M-estimators is either better or as good as z-thresholding to estimate robust CBF maps. In the presence of outliers, Huber's M-estimator is always more accurate than the sample mean.

averaging until $20 \%$ of volumes are corrupted. If more than $20 \%$ of the volumes are affected by outliers, then M-estimators provide better estimates than both z-score thresholding and averaging. The robust M-estimator CBF 
map is closer to the ground truth and less sensitive to an increase in the number of outliers. The same behaviour is observed with a low number of corrupted voxels per volume except that Huber's M-estimator outperforms zthresholding as soon as more than $5 \%$ of the volumes are corrupted (instead of $20 \%$ for medium or high levels of volume corruption). The lower performance of z-thresholding when the number of corrupted volumes exceeds $20 \%$ (or $5 \%$ with low corruption) is a consequence of the masking effect which penalize this estimator when several outliers are present in the series. Moreover, the performance of Huber's M-estimator always depicts a smaller standard error than z-thresholding.

Both Huber's M-estimator and z-score thresholding provide better estimates than the sample mean. As expected from the theory, Huber's Mestimator outperforms z-score thresholding when the number of corrupted volume is large, due to masking effect, or in the presence of a low level of corruption per volume. This latter effect is easily explained by the fact that the method proposed in [1] is based on a global mean and standard deviation estimate per volume (or slice) and is therefore less suited to detect sparsely corrupted volumes.

\subsubsection{Simulation based on pathological data:}

Fig. 7 presents the simulation study based on the second dataset, obtained from a subject diagnosed with a brain tumour. The performances of sample mean, z-score thresholding [1], and Huber's M-estimator are now assessed by measuring the correlation coefficient of ASL CBF with DSC CBF.

The simulation involving a high level of volume corruption leads to very similar results than the one obtained in the previous section on healthy subject data. Both Huber's M-estimator and z-thresholding perform better than averaging until $20 \%$ of the volumes are corrupted. After this threshold, zthresholding performances drop until reaching the same correlation as the sample mean for $30 \%$ of outlier volumes while Huber's M-estimator outperform both alternative approaches. For a medium level of volume corruption, the same tendency is observable. The coherency of these result with the one obtained in the previous section also suggests that correlation with DSC is a good measure of ASL CBF map quality.

With a low level of volume corruption, the trend is less clear. Overall the correlation coefficient seems much less affected by the increasing number of outliers. Z-score thresholding and Huber's M-estimator are both better estimator of the mean than the sample mean. Z-score thresholding however 

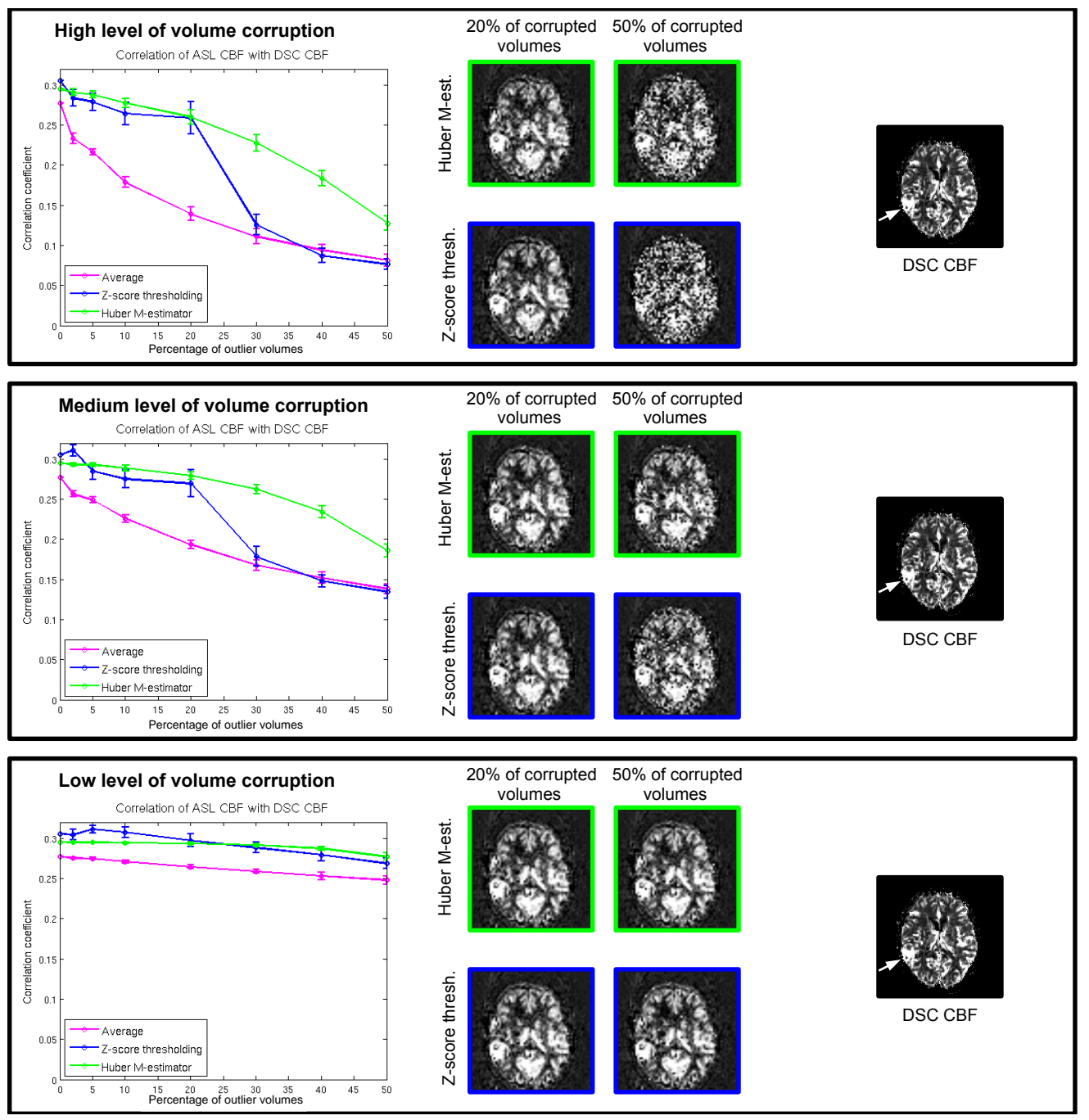

Figure 7: Patient dataset with simulated outliers: correlation of ASL CBF map, computed by M-estimator, z-score thresholding [1] and sample mean, with the DSC CBF map. High, medium and low level of volume corruption, from $0 \%$ to $50 \%$ of corrupted volumes. The white arrow points the tumour site. A similar behaviour as for healthy subject simulation (fig. 6) is observed outlining that correlation with DSC CBF is a valuable indicator to measure the quality of the ASL CBF estimates.

displays a higher variance in its performance estimates. In comparison with the previous simulation study, there is probably a higher level of noise in the so-called "uncorrupted" pathological data than in the "uncorrupted" healthy 
subject data. The inherent higher level of noise in pathological data might prevent the correct detection of low level of volume corruption.

\subsection{Validation on real clinical data}

Full dataset. For each subject, the performances of the sample mean, zscore thresholding, and Huber's M-estimator are assessed by measuring the Pearson linear correlation coefficient of the estimated ASL CBF maps with the DSC CBF map. Table 1 presents the average correlation coefficient obtained across the group of 24 patients in the first column. The average increase in correlation coefficient by comparison to the sample mean for both Huber's M-estimator and z-thresholding is displayed in the second column of table 1.

Overall, the correlation is improved for both Huber's M-estimator and z-score thresholding. While this improvement is significant for Huber's Mestimator (paired t-test $\mathrm{p}=0.01$ ), there is only a tendency for $\mathrm{z}$-score thresholding (paired t-test $\mathrm{p}=0.08$ ). In this dataset, there was no significant difference between the two filtering methods (paired t-test $\mathrm{p}=0.36$ ).

Table 1: Real clinical dataset: Correlation with DSC CBF map of ASL CBF map estimated with the sample mean, z-score thresholding or Huber's M-estimator in 24 patients diagnosed with brain tumours. The average correlation coefficient (column 1) and increase in correlation coefficient with respect to the sample mean (column 2) are reported.

\begin{tabular}{ccc}
\hline & Correlation & Increase w.r.t. average \\
\hline Sample mean & $0.29 \pm 0.09$ & - \\
z-score thresholding & $0.30 \pm 0.09$ & $0.02 \pm 0.05$ \\
Huber M-estimator & $0.31 \pm 0.10$ & $0.02 \pm 0.04$ \\
\hline
\end{tabular}

Fig. 8 presents an example of robust ASL CBF maps in which motion artefacts are significantly reduced by both Huber's M-estimator and z-thresholding.

Comparison based on subsets of the data. Fig. 9 reports the average correlation coefficient (across the group of 24 patients) for datasets with various number or repetitions $(r \in[5,10,20,30,40,50])$. Both z-thresholding and Huber's M-estimator outperform the sample mean for all the number of repetitions studied. Huber's M-estimator appears marginally better than z-thresholding. 

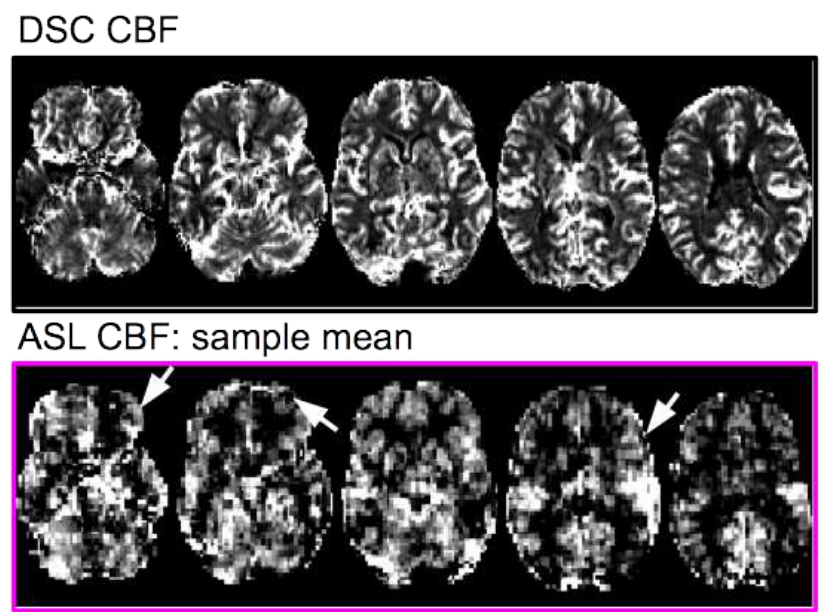

ASL CBF : z-score thresholding

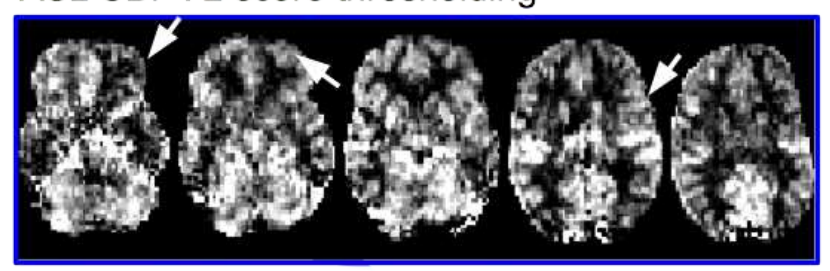

ASL CBF : Huber's M-estimator

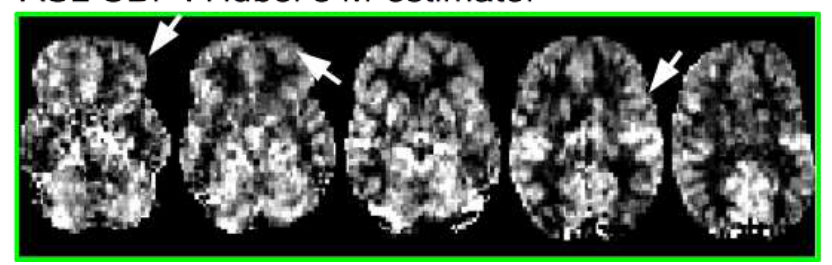

Figure 8: Example of robust CBF map in one patient: six axial slices. White arrows outlines large artefacts present in the averaged perfusion-weighted map and correctly corrected by both z-score thresholding and M-estimator.

Fig. 10 presents an example in which we can clearly see the improved quality of both robust ASL CBF maps.

\section{Discussion and conclusion}

We studied the ability of Huber's M-estimator to compute robust CBF maps in ASL. The behaviour of this estimator was studied in both simulated and real clinical datasets and compared to an outlier removal technique based on z-thresholding previously introduced in the ASL literature [1]. 


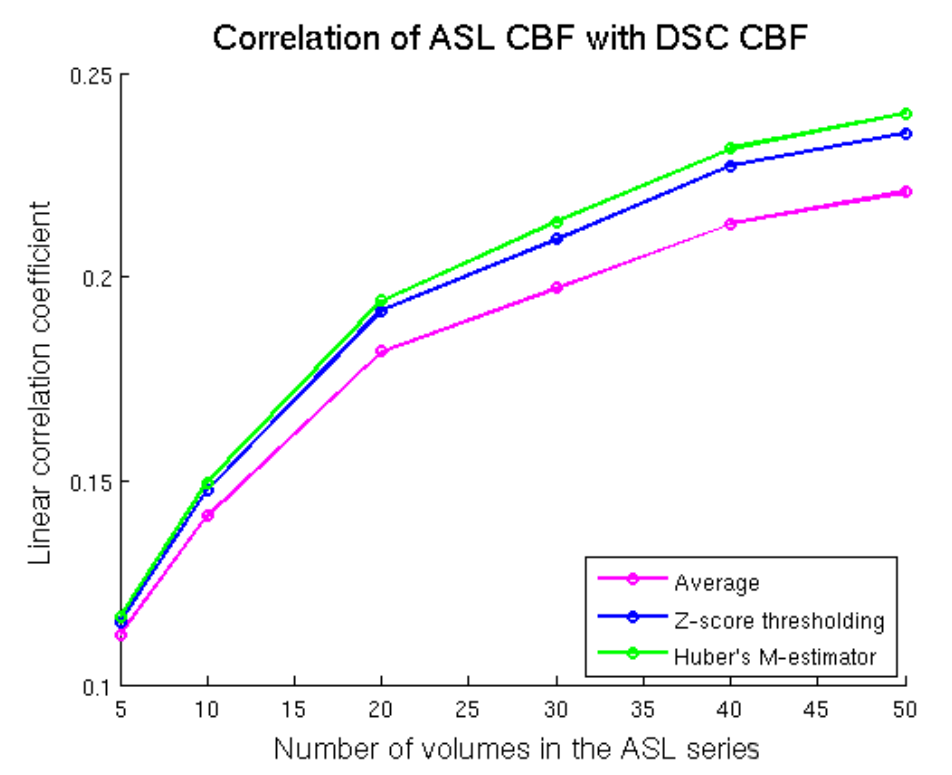

Figure 9: Real clinical dataset: correlation of ASL CBF map with DSC CBF for different number of repetitions $r$.

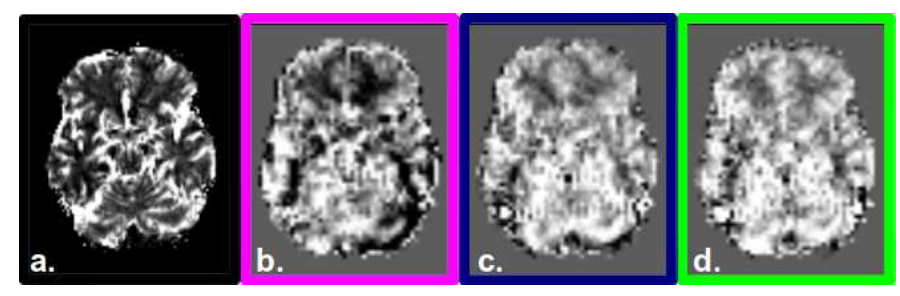

Figure 10: DSC CBF estimate (a) and ASL CBF estimates based on 40 repetitions: sample mean (b), z-thresholding (c), Huber's M-estimator (d).

We demonstrated the superior versatility of Huber's M-estimator by comparison to current practice in ASL, i.e. sample averaging, and state-of-theart, i.e. z-score thresholding from [1]. These results are in line with the known statistical properties of such estimators and outline the shortcomings of z-score thresholding, notably its sensitivity to masking effects.

Out of this study, it is confirmed that outlier filtering, either via outlier removal or M-estimation, provides more robust CBF maps than the sample mean. The simulation study clearly confirmed the superior robustness of M-estimators over z-score thresholding in the presence of outliers. On the tested clinical cases, the superiority was less pronounced but still present. 
The real dataset under study comes from clinical routine examinations and was not especially selected for the purpose of this study. Among the 24 cases, only a subset might be affected by severe artefacts. A comparison of the two robust methods (M-estimators, z-score) on a larger clinical sample would be needed in order to further investigate their respective performances on real data. Overall, both robust methods outperform the sample mean on simulated and real data and Huber's M-estimates are either as good as or better than z-thresholding and are always less variable.

In the context of this paper, we estimated the perfusion maps based on pair-wise subtracted ASL series. In functional ASL, other authors have suggested to work on the complete ASL time-course before subtraction [3]. Likewise, the proposed Huber's M-estimator could be applied on the unsubtracted series. In this case care should be taken in order to deal with temporally correlated noise [17] as opposed to working with the pair-wise subtracted series. Further investigations are needed in order to find out which of the alternative implementation is more appropriate for ASL data.

As M-estimators are able to deal with a broader range of outliers, we recommend the use of M-estimators as robust method to compute ASL CBF maps.

[1] H. Tan, J. A. Maldjian, J. M. Pollock, J. H. Burdette, L. Y. Yang, A. R. Deibler, R. A. Kraft, A fast, effective filtering method for improving clinical pulsed arterial spin labeling MRI., Journal of Magnetic Resonance Imaging 29 (5) (2009) 1134-9.

[2] J. Detre, J. Leigh, D. S. Williams, A. P. Koretsky, Perfusion imaging, Magnetic Resonance in Medicine 23 (1992) 37-45.

[3] J. A. Mumford, L. Hernandez-Garcia, G. R. Lee, T. E. Nichols, Estimation efficiency and statistical power in arterial spin labeling fMRI., NeuroImage 33 (1) (2006) 103-14.

[4] Y. Wang, A. J. Saykin, J. Pfeuffer, C. Lin, K. M. Mosier, L. Shen, S. Kim, G. D. Hutchins, Regional reproducibility of pulsed arterial spin labeling perfusion imaging at 3T., NeuroImage 54 (2) (2011) 1188-95.

[5] J. A. Detre, H. Rao, D. J. Wang, Y. F. Chen, Z. Wang, Applications of arterial spin labeled MRI in the brain., Journal of Magnetic Resonance Imaging 35 (5) (2012) 1026-37. 
[6] E. T. Petersen, I. Zimine, Y.-C. L. Ho, X. Golay, Non-invasive measurement of perfusion: a critical review of arterial spin labelling techniques., The British journal of radiology 79 (944) (2006) 688-701.

[7] K. Sidaros, K. Olofsson, M. J. Miranda, O. B. Paulson, Arterial spin labeling in the presence of severe motion, Journal of Cerebral Blood Flow \& Metabolism 25 (2005) S382-S382.

[8] R. E. Shiffler, Maximum Z Scores and Outliers, The American Statistician 42 (1) (1988) 79-80.

[9] P. Rousseeuw, A. LeRoy, Robust Regression and Outlier Detection, Wiley series in probability and mathematical statistics. Probability and mathematical statistics, Wiley-Interscience, 2003.

[10] P. J. Huber, Robust estimation of a location parameter, The Annals of Mathematical Statistics 35 (1) (1964) pp. 73-101.

[11] C. Warmuth, M. Günther, C. Zimmer, Quantification of Blood Flow in Brain Tumors: Comparison of Arterial Spin Labeling and Dynamic Susceptibility-weighted Contrast-enhanced MR Imaging, Radiology 228 (4) (2003) 523-532.

[12] C. Maumet, P. Maurel, J.-C. Ferré, C. Barillot, Robust Cerebral Blood Flow Map Estimation in Arterial Spin Labeling, in: Proceedings of the Second International Workshop on Multimodal Brain Image Analysis (MBIA), held in conjunction with MICCAI 2012, Nice, France, 2012, pp. 215-224.

[13] W. S. Krasker, R. E. Welsch, Efficient bounded-influence regression estimation, Journal of the American Statistical Association 77 (379) (1982) $595-604$.

[14] G. Zaharchuk, R. Bammer, M. Straka, A. Shankaranarayan, D. C. Alsop, N. J. Fischbein, S. W. Atlas, M. E. Moseley, Arterial spin-label imaging in patients with normal bolus perfusion-weighted MR imaging findings: pilot identification of the borderzone sign., Radiology 252 (3) (2009) 797-807.

[15] R. B. Buxton, L. R. Frank, E. C. Wong, B. Siewert, S. Warach, R. Edelman, A general kinetic model for quantitative perfusion imaging with 
arterial spin labeling., Magnetic Resonance in Medicine 40 (3) (1998) 383-396.

[16] L. Ostergaard, R. M. Weisskoff, D. A. Chesler, C. Gyldensted, B. R. Rosen, High resolution measurement of cerebral blood flow using intravascular tracer bolus passages. Part I: Mathematical approach and statistical analysis., Magnetic Resonance in Medicine 36 (5) (1996) 715725 .

[17] G. K. Aguirre, J. A. Detre, E. Zarahn, D. C. Alsop, Experimental design and the relative sensitivity of BOLD and perfusion fMRI., NeuroImage 15 (3) (2002) 488-500. 